\title{
Institutional Factors Contribute to Variation in Intubation Rates in Status Epilepticus
}

The Neurohospitalist

2019, Vol. 9(3) 133-139

(C) The Author(s) 2018

Article reuse guidelines:

sagepub.com/journals-permissions

DOI: $10.1177 / 1941874418819349$

journals.sagepub.com/home/ $\mathrm{NHO}$

(S)AGE

\author{
Elan L. Guterman, MD', James F. Burke, MD, MS', \\ S. Andrew Josephson, MD', and John P. Betjemann, MD'
}

\begin{abstract}
Background: To explore intubation rates among patients with status epilepticus (SE) and the degree of institutional variation. Methods: Serial cross-sectional study of SE-related hospitalizations from 2004 to 2013 using data from the National Inpatient Sample. The primary outcome was intubation of patients with SE. Multivariable models identified predictors of intubation, institutional variation in intubation rates, and the proportion of variance attributable to individual hospitals. This analysis was repeated using data from 5 states in the State Inpatient Databases (SID). Results: There were II 9337 SE hospitalizations. The overall intubation rate was $32.7 \%$ ( $95 \%$ confidence interval [Cl]: $32.2 \%-33.3 \%)$. There was marked variation in estimated intubation rates, ranging from $2 \%$ to $80 \%$ in the lowest and highest quintile after adjustment. There was somewhat less variability in the SID cohort where quintiles ranged from $10 \%$ to $54 \%$. Those undergoing intubation were more often men and presenting with stroke, intracerebral hemorrhage, central nervous system infection, hyponatremia, and alcohol withdrawal. Urban location (odds ratio [OR]: 3.8, 95\% Cl: 2.7-5.5) and hospitalization at a teaching institution (OR: 3.9, 95\% Cl: I.2-I2.6) were even stronger predictors of intubation after adjustment for clinical factors. A regression including both patient- and hospital-level variables to predict intubation also performed better than a regression including patient factors alone ( $C$ statistic $0.8 \mathrm{I}$ vs 0.59 , respectively). Conclusions: There is considerable institutional variation in intubation rates for SE independent of patient characteristics suggesting that decisions around intubation rest heavily on where one is hospitalized. Further work is needed to clarify how this variation influences outcomes.
\end{abstract}

\section{Keywords}

status epilepticus, health services research, intubation, neurocritical care

\section{Introduction}

Status epilepticus (SE) is a common neurologic emergency. ${ }^{1}$ Medications used for early seizure termination depress mental status and respiratory function and approximately $20 \%$ of patients require endotracheal intubation underscoring the importance of airway management. ${ }^{2-6}$ The decision to intubate is not straightforward and must balance the risks of aspiration and respiratory failure against risks associated with intubation. With little known about how patient factors influence the risk of respiratory depression, practice is dictated by expert opinion and may be subject to considerable institutional variability. ${ }^{7}$

Part of this variability relates to differences in hospital patient population. Older age, refractory seizures, receiving additional rescue antiepileptic medications, underlying stroke, metabolic abnormality, and central nervous system (CNS) tumor are associated with higher rates of intubation. ${ }^{6}$ Yet, there are likely differences in hospital practice that extend beyond differences in patient composition. The degree of hospital variation, institutional characteristics driving this variation, and subsequent impact on outcome are largely unknown.

A better understanding of institutional variation is important because intubation may be associated with higher mortality independent of disease severity. ${ }^{6,8-10}$ Variation also has implications on SE-related costs, which currently approach US\$4 billion annually in the United States. ${ }^{11}$ Using nationally representative data, we sought to determine the overall rate of intubation among patients with SE, determine patient- and hospital-level predictors of intubation, and estimate the degree

\footnotetext{
' Department of Neurology, University of California, San Francisco, CA, USA

${ }^{2}$ Department of Neurology, University of Michigan, Ann Arbor, MI, USA

Corresponding Author:

Elan L. Guterman, 505 Parnassus Avenue, M798 Box 01 14, San Francisco, CA 94143 , USA.

Email: elan.guterman@ucsf.edu
} 
of institutional variation after controlling for individual clinical characteristics.

\section{Materials and Methods}

\section{Study Design}

We performed a retrospective serial cross-sectional study to compare institutional intubation rates among patients hospitalized with SE and determine how patient- and hospital-level characteristics predict intubation use. We used data from the Healthcare Cost and Utilization Project National Inpatient Sample (NIS) and State Inpatient Databases (SID) as well as from the American Hospital Association (AHA) Annual Survey Database. The NIS is a nationally representative data set from a stratified sample of US community hospitals. ${ }^{12}$ In order to ensure the NIS sampling strategy was not influencing our results, we also did an analysis using SID data. The SID contains information from all hospitalizations within a particular state thus there is no sampling used for data collection, but the cohort is more geographically limited. ${ }^{13}$ The AHA is a survey of all hospitals nationally that can be linked to data in SID to provide hospital-level characteristics. We used NIS data from 2004 to 2013. We then repeated the analysis with SID data from Florida, Nebraska, North Carolina, New York, and Utah from 2006 to 2010. The SID cohort was linked to AHA data from 2008 (which was used to provide hospital characteristics for 2006, 2007, and 2008 SID data) to 2010.

\section{Standard Protocol Approvals, Registrations, and Patient Consents}

This study was approved by the institutional review board of University of California, San Francisco and was exempt from the need for informed consent.

\section{Study Cohort}

We included adult patients aged 18 years or older hospitalized with a primary discharge diagnosis of SE using International Classification of Diseases, Ninth Revision (ICD-9) codes for focal SE (ICD-9 345.2) and generalized SE (ICD-9 345.3). The ICD coding for generalized SE was previously validated with a positive predictive value over $80 \%$ in identifying patients with epilepsy. ${ }^{9,14}$ We only included emergency department (ED) admissions to exclude transfer patients where intubation may have occurred previously. We identified ED admissions through codes for admission source, ED revenue, ED services, and ED-specific procedures.

The NIS and SID capture hospital encounters rather than unique patients. Individuals with multiple hospitalizations could be included in our analysis cohort multiple times. Hospitalizations for cardiac arrest were excluded because the proximate reason for intubation would be ambiguous.

\section{Study Outcomes and Measurements}

The primary outcome was intubation at any point during hospitalization. Intubation was identified using $I C D-9$ procedure codes for intubation (ICD-9 96.04) and mechanical ventilation (ICD-9 96.7x) which together carry a sensitivity over $80 \%$ in identifying patients mechanically ventilated at any point during hospitalization. ${ }^{9,15,16} \mathrm{We}$ included "other intubation of the respiratory tract" (ICD-9 96.05) to increase sensitivity.

Patient age, sex, race, insurance payer, palliative care, medical comorbidities, and potential etiology underlying SE were included in all multivariable models. The SE etiology was used as a marker of severity based on data demonstrating an association between seizure etiology and mortality. ${ }^{2,10}$ We identified potential seizure etiology through discharge diagnosis codes. This included codes for ischemic stroke (ICD-9 433.x1, 434.x1, 436), intracerebral hemorrhage (ICD-9 431.x), CNS tumor (ICD-9 191x, 239.6, 198.3), traumatic brain injury (ICD-9 800.x-804.x, 850.x), meningoencephalitis (ICD-9 320.x-323.x), hyponatremia (ICD-9 276.1), and alcohol withdrawal (ICD-9 291.81). ${ }^{17-22}$ We identified palliative care patients using $I C D-9$ V66.7. ${ }^{21}$ We also identified nonepileptic convulsions using discharge diagnosis codes for conversion disorder (ICD-9 300.1) and "other convulsions" (ICD-9 780.39). ${ }^{23}$ Charlson Comorbidity Index was used as a marker of severity. ${ }^{24,25}$

The NIS hospital-level variables included hospital region, location (urban, rural), academic status, size (small, medium, large based upon bed number, hospital location, and academic standing), and the interaction of hospital location and academic status. Region was classified according to US Census Bureau regions (Northeast, Midwest, South, West). ${ }^{12}$ The SID hospital-level variables did not include hospital region because the sets themselves are specific to a single state. We defined a teaching hospital variable using the same method employed by NIS. We used hospital bed number rather than the categorical variable hospital size.

\section{Statistics}

For descriptive statistics, Pearson $\chi^{2}$ test compared categorical variables and $t$ test compared continuous variables. For multivariate analysis, we examined patient- and hospital-level predictors of intubation. Because patient care is correlated within a given institution, we used a multilevel mixed effects model that included a random hospital-level intercept to account for clustering within an institution. We added survey weights to account for the NIS sampling strategy for the univariate and multivariate analyses.

Because our goal was to characterize institutional variation nationally, we used the NIS data for our primary analysis. Using this data, we compared predictiveness of 2 multivariable regression models using $\mathrm{C}$ statistics. The first model exclusively used patient-level covariates to predict intubation use while the second model added hospital-level covariates. 
We next calculated differences in the predicted rates of intubation for urban as opposed to rural and teaching as opposed to nonteaching hospitals using average marginal effects that were adjusted for differences in underlying seizure etiology. We calculated the proportion of variance at the hospital level using the intraclass correlation coefficient. We then used our mixed effects model to estimate the range of intubation rates across hospitals nationally.

We subsequently performed a series of sensitivity analyses. We directly calculated the range of hospital intubation rates and repeated the primary analyses using SID data. This allowed us to analyze all (rather than a sample of) acute hospitalizations but only for a limited number of states. Because some variability may reflect differences in hospital patient population, we also repeated the NIS analysis after isolating patients presenting to urban hospitals and teaching hospitals where the patient populations are more similar across institutions. We also performed subgroup analyses excluding patients with focal SE and possible nonepileptic convulsions who typically do not require ventilatory assistance because differences in hospital volume of these patients could influence our results. Finally, NIS changed its sampling strategy in 2012 (initially including all discharges from $20 \%$ of US community hospitals and after 2012 , including $20 \%$ of discharges from all US community hospitals); thus, we performed a sensitivity analysis excluding discharges after 2011. Statistical analyses were performed using Stata (Version 14, StataCorp, College Station, Texas).

\section{Results}

\section{Patient Characteristics and Intubation}

We included 199337 (24 831 unweighted) hospitalizations for SE in the analysis. Intubation occurred in 39072 (95\% confidence interval [CI]: 38 381-39 765) admissions, yielding an overall intubation rate of $32.7 \%$ (95\% CI: $32.2 \%-33.3 \%$ ). The intubation rate increased from $28.8 \%$ (95\% CI: $27.1 \%-30.5 \%$ ) in 2008 to $37.7 \%$ (95\% CI: 36.0\%-39.5\%) in 2015 (Figure 1). Intubation varied by insurance status and intubated patients were more often men, receiving palliative care, and hospitalized with stroke, intracerebral hemorrhage, CNS infection, hyponatremia, and alcohol withdrawal (Table 1).

After adjusting for patient-level variables, women were still less likely to undergo intubation (odds ratio [OR]: 0.8 , 95\% CI: 0.8-0.9). The odds of intubation were the lowest for those with Medicare insurance as compared to Medicaid, private, self-pay, no charge, and other health insurance providers. The associations between intubation and palliative care (OR: 1.4, 95\% CI: 1.1-1.8), stroke (OR: 2.4, 95\% CI: 1.9-3.1), intracerebral hemorrhage (OR: 3.5, 95\% CI: 1.9-6.3), CNS infection (OR: 2.3, 95\% CI: 1.7-3.2), hyponatremia (OR: 1.4, 95\% CI: 1.2-1.5), and alcohol withdrawal (OR: 1.9, 95\% CI: 1.6-2.3) also persisted after adjustment (Table 2).

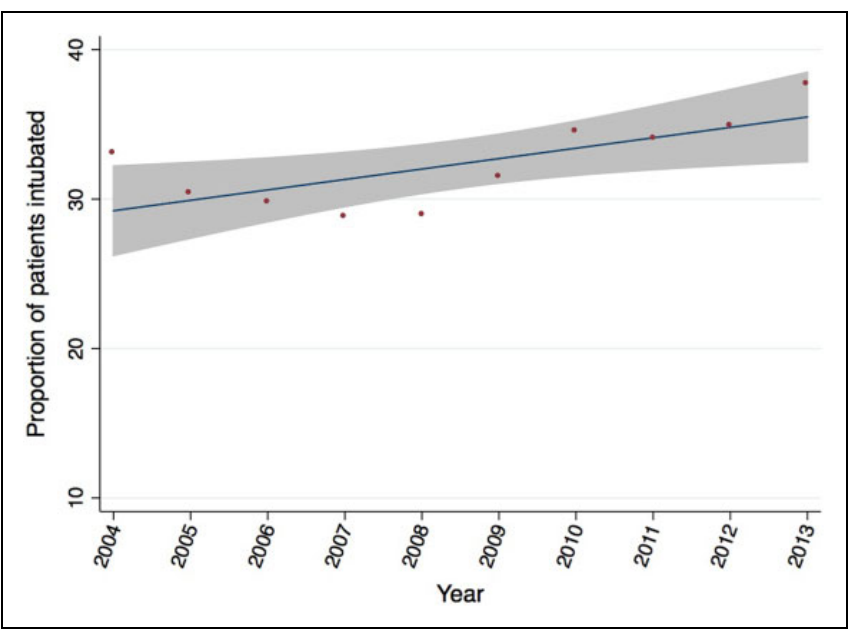

Figure I. Intubation among patients with status epilepticus over time, 2004 to 2013 . Changes in the rate of intubation over time. Dots represent the total intubation rate across all hospitals for a given year, the line represents the predicted trend with the $95 \%$ confidence interval (shaded area).

\section{Hospital Characteristics and Intubation}

Teaching hospitals (OR: 4.4, 95\% CI: 1.4-14.0) and urban location (OR: 3.8, 95\% CI: 2.6-5.4) were both strongly correlated with intubation. There were higher odds of intubation in the Northeast as compared to the Midwest (OR: 0.6, 95\% CI: 0.4-0.8) and South (OR: 0.8, 95\% CI: 0.6-1.0; Table 2).

Model predictiveness increased from the patient-level model (C statistic 0.59) to the model incorporating institutional factors (C statistic 0.81 ). The intraclass correlation coefficient was 0.66 (95\% CI: 0.63-0.69) demonstrating clustering at the hospital level. Hospital intubation rates ranged from an estimated $2 \%$ in the lowest quintile to $80 \%$ in the highest quintile. We examined whether there was persistent variation after isolating the analysis to hospital subgroups (teaching only, nonteaching only, urban only, rural only) and found that there continued to be a wide range of estimated intubation rates among urban hospitals, teaching hospitals, and nonteaching hospitals, while there was less variation among nonurban hospitals (Figure 2). The marginal effects analysis showed the probability of intubation was 9 percentage points higher in urban hospitals as compared nonurban hospitals and 7 percentage points higher for teaching as compared to nonteaching hospitals. This analysis excluded patients who had an underlying seizure etiology associated with intubation (stroke, intracerebral hemorrhage, CNS infection, hyponatremia, and alcohol withdrawal) but did not account for other patientlevel variables.

Our subgroup analyses excluding patients with focal SE and nonepileptic convulsions recapitulated our results. Excluding hospitalizations after 2011, when the NIS changed its sampling strategy, did not affect results of the regression or model $\mathrm{C}$ statistics. The intraclass correlation coefficient did, however, decrease to 0.47 (95\% CI: 0.43-0.51). 
Table I. Baseline Characteristics Among Patients Admitted With Status Epilepticus.

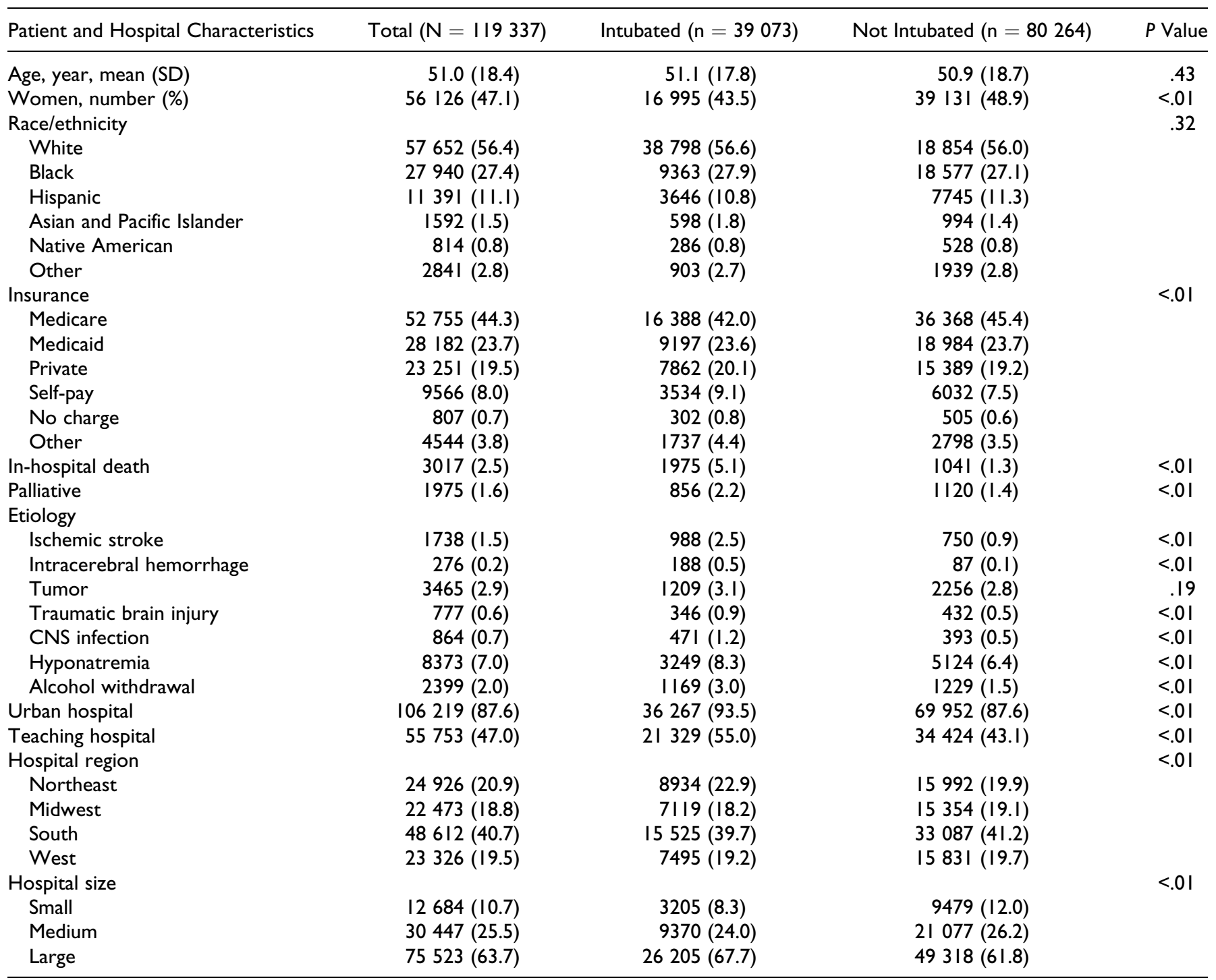

Abbreviations: CNS, central nervous system; COPD, chronic obstructive pulmonary disease.

${ }^{a}$ All values represent survey weighted counts.

Repeating the primary analysis using our SID data showed similar baseline characteristics (Table S1). The overall intubation rate was $31.6 \%$, similar to the NIS cohort, though the range of intubation rates was narrower. We did not see the same difference in intubation across insurance status in our univariate analysis but the multivariate analysis again demonstrated higher odds of intubation among patients with Medicaid (OR: 1.4, 95\% CI: 1.2-1.6), self-pay (OR: 1.3, 95\% CI: 1.1-1.5), and other insurance (OR: 1.3, 95\% CI: 1.0-1.6). Those who were not charged did not have different odds of intubation compared to those with Medicare for insurance but this subgroup was small (Table S1; Table S2). The SID states combined together had hospital intubation rates ranging from $10 \%$ to $54 \%$. Teaching hospitals (OR: $2.3,95 \%$ CI: $1.3-3.9$ ) and urban location (OR: 1.6, 95\% CI: 1.2-2.3) continued to be correlated with intubation and model predictiveness again increased from the patient-level model (C statistic 0.60 ) to the model incorporating institutional factors (C statistic 0.72 ; Table S2).

\section{Discussion}

We found that intubation rates for patients with SE are increasing over time and vary markedly across hospitals. Intubation is scarcely performed at hospitals in the lowest intubation quintile ( $2 \%$ NIS and $10 \%$ SID), while it is very common in the highest quintile ( $80 \%$ NIS and $54 \%$ SID). Intubation practice is less variable in rural settings but urban, teaching, and nonteaching hospitals continue to show wide variation in use of intubation for SE. Hospital characteristics such as urban 
Table 2. Comparison Between Patient-Level Logistic Regression and Mixed Effects Model Predicting Intubation in Status Epilepticus.

\begin{tabular}{|c|c|c|c|c|}
\hline \multirow{3}{*}{$\begin{array}{l}\text { Patient and Hospital Characteristics } \\
\text { C-statistic }\end{array}$} & \multicolumn{2}{|c|}{ Model I } & \multicolumn{2}{|c|}{ Model 2} \\
\hline & 0.59 & & 0.80 & \\
\hline & OR & $95 \% \mathrm{Cl}$ & OR & $95 \% \mathrm{Cl}$ \\
\hline Urban hospital & & & 3.8 & 2.6-5.4 \\
\hline Teaching hospital & & & 3.9 & $1.2-12.6$ \\
\hline \multicolumn{5}{|l|}{ Hospital region (Reference: Northeast) } \\
\hline Midwest & & & 0.6 & 0.4-0.8 \\
\hline South & & & 0.8 & $0.6-1.0$ \\
\hline West & & & 0.8 & $0.6-1.0$ \\
\hline \multicolumn{5}{|l|}{ Hospital size (Reference: Small) } \\
\hline Medium & & & 1.6 & I.2-2.2 \\
\hline Large & & & 2.0 & I.5-2.7 \\
\hline Age, years, mean & 1.0 & I.0-I.0 & 1.0 & $1.0-1.0$ \\
\hline Women, number (\%) & 0.8 & $0.8-0.9$ & 0.8 & $0.8-0.9$ \\
\hline \multicolumn{5}{|l|}{ Insurance (Reference: Medicare) } \\
\hline Medicaid & 1.2 & I.I-I.3 & I.I & $1.0-1.2$ \\
\hline Private & 1.2 & I.I-I.3 & 1.2 & I.I-I.4 \\
\hline Self-pay & 1.4 & $1.3-1.6$ & 1.4 & $1.2-1.7$ \\
\hline No charge & 1.4 & $1.0-2.0$ & 1.3 & $0.8-1.9$ \\
\hline Other & $\mathrm{I} .4$ & I.2-I.6 & 1.3 & I.I-I.6 \\
\hline Palliative care & $\mathrm{I} .4$ & I.I-I.7 & 1.3 & I.0-I.7 \\
\hline \multicolumn{5}{|l|}{ Etiology } \\
\hline Stroke & 2.3 & $1.9-3.0$ & 2.7 & $2.0-3.8$ \\
\hline Intracerebral hemorrhage & 3.5 & 1.9-6.3 & 3.6 & I.6-7.8 \\
\hline Tumor & 0.9 & $0.7-1.1$ & 0.8 & $0.6-1.0$ \\
\hline Traumatic brain injury & 1.3 & $0.9-1.8$ & 1.4 & $0.9-2.2$ \\
\hline CNS infection & 2.3 & I.6-3.I & 2.2 & I.5-3.4 \\
\hline Hyponatremia & $\mathrm{I} .4$ & $1.2-1.5$ & 1.5 & $1.3-1.7$ \\
\hline Alcohol withdrawal & 1.9 & I.6-2.3 & 2.1 & I.6-2.7 \\
\hline
\end{tabular}

Abbreviations: $\mathrm{Cl}$, confidence interval; CNS, central nervous system; NIS, National Inpatient Sample; OR, odds ratio.

aModels also adjusted for race and Charlson Comorbidity Index. Hospitallevel model also adjusted for the interaction between teaching and urban hospital variable.

${ }^{\mathrm{b}}$ The NIS survey weights applied to both models.

${ }^{\mathrm{c}}$ Bold font indicates statistically significant with a $P$ value $<.05$.

${ }^{\mathrm{d} P} P$ value $C$-statistic $<.01$.

location and academic standing were strong predictors of intubation after adjusting for patient-level predictors. Similarly, our intraclass correlation demonstrated that intubation decisions were clustered by hospital. These findings may in part be explained by unmeasured differences in hospital patient population but this likely cannot account for the magnitude of variation, suggesting that where one is hospitalized may alter your likelihood of intubation considerably. This is important because intubation itself may affect patient outcome in which case we must better define the appropriate clinical indications for intubation in order to inform safer, more effective airway management. ${ }^{26-28}$

We found an overall intubation rate of $33 \%$ at some point during hospitalization. This rate is higher than previous national estimates, which come from prehospital and ED treatment trials for SE and may not include a representative set of hospitals. ${ }^{4-6}$ In prehospital treatment trials, appropriately dosed benzodiazepines and cessation of SE prior to ED arrival resulted in lower rates of intubation. ${ }^{3,6}$ The higher rate of intubation in our study may reflect nonstandardized prehospital treatment resulting in more patients with inadequate seizure control upon ED arrival. We also likely captured prehospital intubations in our study population because hospital mechanical ventilation codes are still used. This higher intubation rate may provide a more accurate national representation of SE management, serving as a preliminary benchmark for hospitals to examine their own quality of care.

Rates of intubation also increased over the study period. A landmark prehospital SE trial showed a trend toward lower rates of respiratory depression among patients receiving benzodiazepines as compared to placebo. More widespread prehospital benzodiazepine use would theoretically lower, as oppose to increase, intubation which is at odds with our findings. ${ }^{3}$ This may again reflect nonstandardized prehospital treatment.

Individual factors that increased odds of intubation included male sex and presenting with stroke, intracerebral hemorrhage, CNS infection, hyponatremia, and alcohol withdrawal. The higher risk of ventilatory failure may reflect the underlying illnesses as well as the response to pharmacologic treatment. ${ }^{9,29}$ In addition, odds of intubation were higher among patients with Medicaid, private, and self-pay health insurance in the multivariable analyses of both our NIS and SID cohorts. This was true after adjusting for care at an individual hospital thus likely reflects differences in the epilepsy patient population covered by each insurance type but it remains unclear whether it reflects differences in the underlying cause of epilepsy across insurance groups, differing epilepsy management, or a combination of both factors.

Differences in hospital patient population did not adequately explain differences in intubation rates across institutions. Hospital location and academic standing were stronger predictors of intubation than the patient-level covariates. After adjustment, the likelihood of intubation among patients presenting to a teaching hospital and urban hospital were, respectively, 7 and 9 percentage points higher than their nonacademic and rural counterparts. Adding hospital-level covariates to the regression model improved our ability to predict intubation considerably. This is likely in part explained by unmeasured differences in the patient populations. Academic and urban centers may care for a higher proportion of patients with refractory and superrefractory SE whose care often requires intubation for treatment with intravenous anesthetic medications and has been associated with both higher mean ventilation time and cost of care. ${ }^{26,30}$ Highvolume emergency departments also may have more difficulty monitoring patients closely, translating to lower thresholds for intubation to provide consistent ventilatory assistance. Alternatively, variation in prehospital care may also contribute to this variation. Yet, there was considerable variation in intubation rates after restricting the analysis to urban hospitals, teaching hospitals, and nonteaching hospitals in isolation 


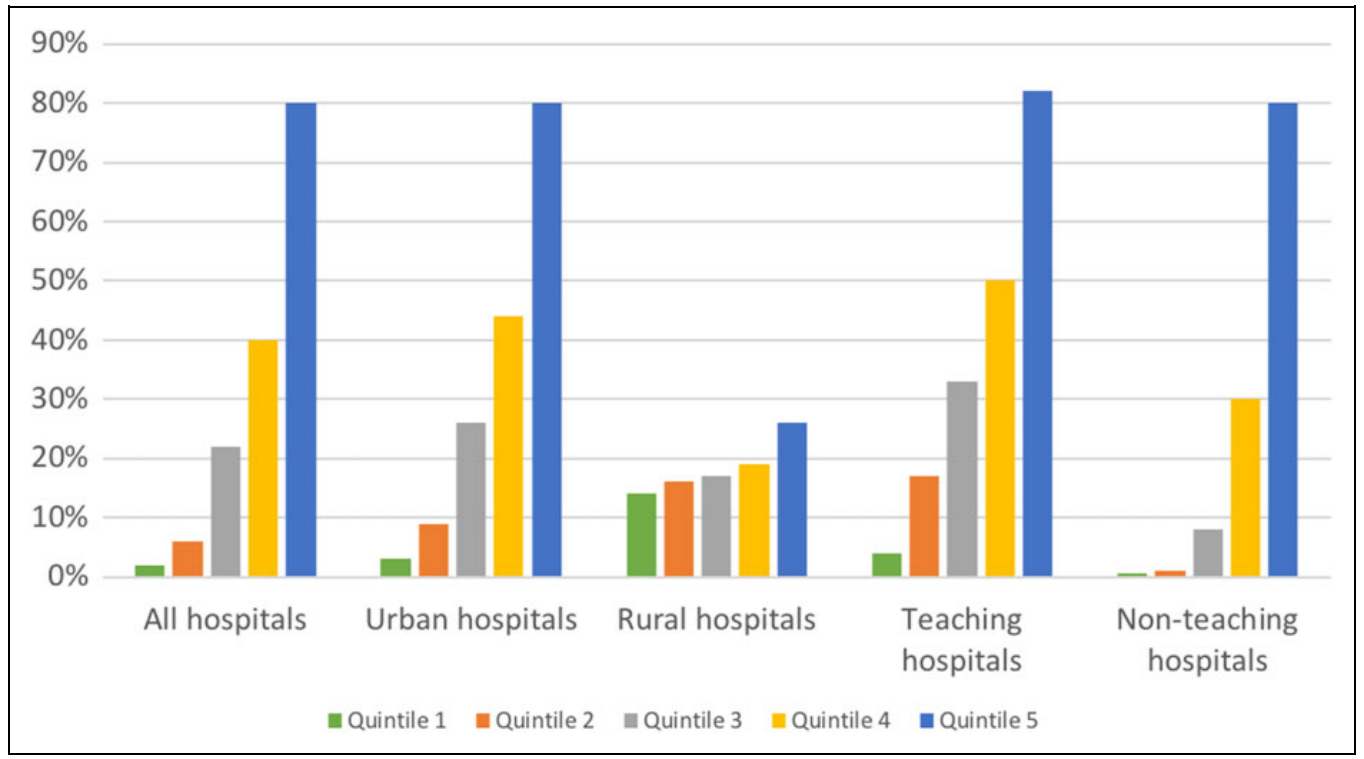

Figure 2. Predicted rates of intubation. Estimated intubation rates across hospital quintiles calculated from the mean odds ratio of our fixed effects and our random hospital-level intercept. The analysis was performed for all hospitals as well as hospital subgroups (urban hospitals, rural hospitals, teaching hospitals, nonteaching hospitals) to better account for differences in patient population. This demonstrates how intubation rates vary when holding all patient factors equal.

making it unlikely that unmeasured patient factors are solely responsible for the observed variance.

These findings draw attention to the limitations of our study. Our data do not include important potential confounders including SE duration, Glasgow Coma Scale, medications used for treatment, and treating physician specialty. To limit confounding, we include information about potential seizure etiology and disease comorbidities, both correlated with SE severity and outcome; however, we do not know the exact relationship between potential SE etiology and seizure. We also excluded patients with cardiac arrest where intubation is nearly expected. As with all large database studies, we are also prone to case ascertainment and measurement errors in developing our cohort. Previous studies demonstrate that the positive predictive value of $I C D-9$ coding for epilepsy is excellent ( $>97 \%)$ and for generalized SE is good ( $>80 \%)$; however, validation for $I C D-9$ coding of diseases underlying seizure (stroke, hyponatremia, etc) is variable and at times lacking. ${ }^{31}$ Intubation codes also identify mechanical ventilation at any point during hospitalization. This includes prehospital intubation where patients are mechanically ventilated after arrival and patients intubated for diseases unrelated to SE. To limit the possibility of the latter, we restricted our analysis to patients with a primary discharge diagnosis of SE for whom seizures and antiepileptic treatment should be contributing directly to the need for ventilatory support, even if additional illnesses are playing a role. Lastly, the sampling strategy used by NIS could in theory limit the reliability of intubation rate calculations, especially after 2012 when NIS used a sample of discharges from all community hospitals in the United States rather than all discharges from a sample of hospitals. To address this potential limitation, we both repeated the analysis excluding hospitalizations after 2012 and repeated the analysis using SID data which provides a complete set of hospitalizations for every state included.

\section{Conclusions}

There is considerable institutional variation in the use of intubation for patients with SE. Intubation also represents one of many clinical decisions in the management of SE where there may be differences in care across hospitals. Further work is needed to understand the relationship between institutional variability and clinical outcome. Identifying which patients benefit from intubation would inform clinical guidelines and allow us to build better systems of care for SE management nationally.

\section{Declaration of Conflicting Interests}

The authors declared no potential conflicts of interest with respect to the research, authorship, and/or publication of this article.

\section{Funding}

The authors disclosed receipt of the following financial support for the research, authorship, and/or publication of this article: This publication was supported by the National Center for Advancing Translational Sciences, National Institutes of Health, through UCSF-CTSI Grant Number UL1 TR001872. Its contents are solely the responsibility of the authors and do not necessarily represent the official views of the NIH. Dr Burke has received grant funding from the National Institute of Neurological Disorders and Stroke through grant numbers K08 NS082597 and R01 MD008879 for investigatorinitiated research. Dr Josephson has received personal fees from 
JAMA Neurology for serving as editor and personal fees from Continuum Audio for serving as interviewer and section editor. Dr Betjemann has received personal fees from JAMA Neurology for serving as web editor.

\section{Supplemental Material}

Supplemental material for this article is available online.

\section{References}

1. DeLorenzo RJ, Hauser WA, Towne AR, et al. A prospective, population-based epidemiologic study of status epilepticus in Richmond, Virginia. Neurology. 1996;46(4):1029-1035.

2. Lowenstein DH, Alldredge B. Status epilepticus. $N$ Engl J Med. 1998;338(14):970-976.

3. Alldredge BK, Gelb AM, Isaacs SM, et al. A comparison of lorazepam, diazepam, and placebo for the treatment of out-ofhospital status epilepticus. N Engl J Med. 2001;345(9):631-637.

4. Silbergleit R, Durkalski V, Lowenstein D, et al. Intramuscular versus intravenous therapy for prehospital status epilepticus (RAMPART). N Engl J Med. 2012;366(7):591-600.

5. Chamberlain JM, Okada P, Holsti M, et al. Lorazepam vs diazepam for pediatric status epilepticus. JAMA. 2014;311(16): 1652-1660.

6. Vohra TT, Miller JB, Nicholas KS, et al. Endotracheal intubation in patients treated for prehospital status epilepticus. Neurocrit Care. 2015;23(1):33-43.

7. Brophy GM, Bell R, Claassen J, Mendelsohn FA, Mayer SA. Guidelines for the evaluation and management of status epilepticus. Neurocrit Care. 2012;17(1):3-23.

8. Claassen J, Lokin JK, Fitzsimmons BF, et al. Predictors of functional disability and mortality after status epilepticus. Neurology. 2002;58(1):139-142.

9. Betjemann JP, Josephson SA, Lowenstein DH, Burke JF. Trends in status epilepticus-related hospitalizations and mortality: redefined in US practice over time. JAMA Neurol. 2015;72(6):650-655.

10. Towne AR, Pellock JM, Ko D, DeLorenzo RJ. Determinants of mortality in status epilepticus. Epilepsia. 1994;35(1):27-34.

11. Penberthy LT, Towne A, Garnett LK, Perlin JB, DeLorenzo RJ. Estimating the economic burden of status epilepticus to the health care system. Seizure. 2005;14(1):46-51.

12. NIS Description of Data Elements. Healthcare Cost and Utilization Project (HCUP). August 2018. Agency for Healthcare Research and Quality, Rockville, MD. http://www.hcupus. ahrq.gov/db/nation/nis/nisdde.jsp. Accessed December 2018.

13. HCUP Central Distributor SID Availability of Data Elements by Year. Healthcare Cost and Utilization Project (HCUP). October 2018. Agency for Healthcare Research and Quality, Rockville, MD. www.hcup-us.ahrq.gov/db/state/siddist/siddist_ddeavailby year.jsp. Accessed December 2018.

14. Jette N, Reid AY, Quan H, Hill MD, Wiebe S. How accurate is ICD coding for epilepsy? Epilepsia. 2010;51(1):62-69.
15. Quan H, Parsons GA, Ghali WA. Validity of procedure codes in international classification of diseases, 9th revision, clinical modification administrative data. Med Care. 2004;42(8): 801-809.

16. Walkey AJ, Wiener RS. Use of noninvasive ventilation in patients with acute respiratory failure, 2000-2009: a population-based study. Ann Am Thorac Soc. 2013;10(1):10-17.

17. Tirschwell DL, Longstreth WT. Validating administrative data in stroke research. Stroke. 2002;33(10):2465-2470.

18. Goldstein LB. Accuracy of ICD-9-CM coding for the identification of patients with acute ischemic stroke: effect of modifier codes. Stroke. 1998;29(8):1602-1604.

19. Prabhakaran S, Herbers P, Khoury J, et al. Is prophylactic anticoagulation for deep venous thrombosis common practice after intracerebral hemorrhage? Stroke. 2014;46(2):369-375.

20. Janus TJ. Coding table. Continuum (Minneap Minn). 2010;16(5 Multiple Sclerosis):243-246.

21. Bazarian JJ, Mcclung J, Shah MN, Cheng YT, Flesher W, Kraus J. Mild traumatic brain injury in the United States, 1998-2000. Brain Inj. 2005;19(2):85-91.

22. Qureshi AI, Adil MM, Suri MFK. Rate of utilization and determinants of withdrawal of care in acute ischemic stroke treated with thrombolytics in USA. Med Care. 2013;51(12):1094-1100.

23. Jette N, Beghi E, Hesdorffer D, et al. ICD coding for epilepsy: past, present, and future - a report by the International League Against Epilepsy Task Force on ICD codes in epilepsy. Epilepsia. 2015;56(3):348-355.

24. Charlson ME, Pompei P, Ales KL, MacKenzie CR. A new method of classifying prognostic comorbidity in longitudinal studies: development and validation. J Chronic Dis. 1987; 40(5):373-383.

25. Alvarez V, Januel JM, Burnand B, Rossetti AO. Role of comorbidities in outcome prediction after status epilepticus. Epilepsia. 2012;53(5):e89-e92.

26. Kortland LM, Alfter A, Bähr O, et al. Costs and cost-driving factors for acute treatment of adults with status epilepticus: a multicenter cohort study from Germany. Epilepsia. 2016; 57(12):2056-2066.

27. Sutter R, Marsch S, Fuhr P, Kaplan PW, Rüegg S. Anesthetic drugs in status epilepticus: risk or rescue? A 6-year cohort study. Neurology. 2014;82(8):656-664.

28. Roppolo LP, Walters K. Airway management in neurological emergencies. Neurocrit Care. 2004;1(4):405-414.

29. Lowenstein DH, Alldredge BK. Status epilepticus at an urban public hospital in the 1980s. Neurology. 1993;43(3 Pt 1): 483-488.

30. Misra UK, Kalita J, Bhoi SK, Dubey D. Cost of status epilepticus in a tertiary care hospital in India. Seizure. 2015;31:94-98.

31. Jetté N, Reid AY, Quan H, Hill MD, Wiebe S. How accurate is ICD coding for epilepsy? Epilepsia. 2010;51(1):62-69. 